\title{
Analysis of Visible Light Communication Link's Performance in Fire Smoke Environment
}

\author{
Yu-Feng Yang $\mathbb{D},{ }^{1,2}$ Kang-Kang Chang $\mathbb{D}^{1}$, and Ying $\mathrm{Ci}^{3}$ \\ ${ }^{1}$ School of Automation \& Information Engineering, Xi'an University of Technology, Xi'an 710048, China \\ ${ }^{2}$ Shaanxi Civil-Military Collaboration Key Laboratory of Intelligence Coordination Networks, Xi'an University of Technology, \\ Xi'an 710048, China \\ ${ }^{3}$ Beijing Institute of Tracking and Communication Technology, Beijing 100000, China
}

Correspondence should be addressed to Kang-Kang Chang; 1427373089@qq.com

Received 9 April 2021; Accepted 20 May 2021; Published 26 May 2021

Academic Editor: Heng Liu

Copyright (c) $2021 \mathrm{Yu}$-Feng Yang et al. This is an open access article distributed under the Creative Commons Attribution License, which permits unrestricted use, distribution, and reproduction in any medium, provided the original work is properly cited.

\begin{abstract}
This paper mainly studies the communication performance of visible light communication link in fire smoke environment. Compared with the previous studies, this study no longer only considers a single wavelength and considers the influence of smoke and background light noise. Firstly, the variation of background light noise caused by sunlight during a day was analyzed, then signal-to-noise ratio (SNR) and bit error rate (BER) of the visible light communication link in fire smoke environment with different visibility were calculated, and finally, the variation of received power with the angle $\beta$ between the LED optical axis and the communication link (from LED to receiver) was analyzed. The results show that, during a day from 07:00 to 18:30, background light noise first increases and then decreases, BER changes in the same trend and reaches the maximum at about 13:00, while SNR changes in the opposite trend and reaches the minimum at about 13:00. When visibility is $100 \mathrm{~m}$, the maximum difference of SNR between 7:00 and 13:00 is about 19 dB, and the maximum difference of BER is about 5.3E-10. At 7:00, the maximum difference of SNR is about $13 \mathrm{~dB}$ between the visibility of $1 \mathrm{~m}$ and the visibility of $100 \mathrm{~m}$, and the maximum difference of BER is about $1.44 E-11$, and received power decreases with the increase of $\beta$. In addition, SNR increases with visibility of fire smoke environment, and BER changes in the opposite trend. Lastly, in order to enhance the performance of visible light communication in fire smoke environment, this paper gives the transmitting power scheme.
\end{abstract}

\section{Introduction}

Currently, the application of visible light communication (VLC) is becoming more and more widespread. The new visible light communication technology based on LED has many advantages: it can meet high-speed communication while realizing lighting, is harmless to human eye health and does not cause radiation damage to the human body, is not easily subject to electromagnetic interference, and has good confidentiality. In addition, VLC technology also does not require radio spectrum certification, which can effectively solve the problem of spectrum limitation of radio frequency (RF) technology [1] and can also be used for indoor target localization. Therefore, this technology is playing an increasingly important role in vehicle networking, underground mines, and other fields.
Although visible light communication has many advantages, it is easily affected by the atmospheric environment [2], and indoor fire smoke and outdoor fire smoke are one of the most important factors. Due to the short light wavelength and serious light scattering, the visible light communication link is easily blocked. In addition, the outdoor rain, fog, snow, and background light noise are also important factors to interfere with the visible light communication link. In order to improve the performance of visible light communication in a special environment, it is necessary to study the transmission characteristic of the visible light communication link in this environment. In 2013, Zhu et al. [3] studied the effect of smoke on free-space optical (FSO) communication and proposed that the attenuation coefficient decreases exponentially as the volume of the shell increases. In 2016, Chen et al. [4] studied the 
transmittance of laser at different wavelengths in smoke. In a $4.8 \mathrm{~m}$ flue, the transmittance of $10.6 \mu \mathrm{m}$ laser is twice that of $1.06 \mu \mathrm{m}$ laser when the smoke concentration is less than $1.0947 \mathrm{~g} / \mathrm{m}^{3}$. In the same year, it was also concluded that, in a $400 \mathrm{~m}$ flue, the transmittance of $10.6 \mu \mathrm{m}$ laser is up to 104 times that of $1.06 \mu \mathrm{m}$ laser [5]. In the same year, Wang and Duan [6] proposed that, in different concentrations of smoke environment, due to the absorption of the laser beam by suspended particles, the laser is attenuated in the process of transmission. In 2019, Jiang and Song [7] proposed that the FMCW laser ranging system is attenuated and absorbed by smoke particles when measuring the echo signal in smoke environment, and this leads to the result that it is difficult to extract effective signals.

However, the above literature studies mainly studied lasers with a certain wavelength, and current research related to visible light communication is less likely to consider environmental factors. There are even fewer research and reports on the communication performance of visible light in fire smoke environment. In this paper, we focus on analyzing the performance of visible light communication link in fire smoke environment and provide a theoretical basis for the application of visible light communication in this environment in the future.

\section{Analysis of Background Light Noise}

The background light noise $P_{b g}$ cannot be ignored in visible light communication in fire smoke environment, and the blackbody radiation model can be used to describe spectral radiance $W\left(\lambda, T_{B}\right)[8,9]$ :

$$
W\left(\lambda, T_{B}\right)=\frac{2 \pi h_{p} c^{2}}{\lambda^{5}}\left[\frac{1}{e^{\left(h_{p} c / \lambda k T_{B}\right)}-1}\right],
$$

where $h p$ is Planck's constant, $c$ is the speed of light, $\lambda$ is the wavelength, $k$ is Boltzmann's constant, and $T_{B}$ is the average surface temperature of the sun, usually taken as $6000 \mathrm{~K}$.

The global solar irradiance $E_{\text {global }}$ has a wavelength range between $0.3 \mu \mathrm{m}$ and $4.0 \mu \mathrm{m}$, and it serves as the input for global spectral irradiance measurements. The peak spectral irradiance $S_{\text {peak }}$ is given by [8]

$$
S_{\text {peak }}=0.0001 E_{\text {global }}^{2}+1.5768 E_{\text {global }} \text {. }
$$

The global solar irradiance $W_{\text {approx }}$ can be derived as [8]

$$
W_{\text {approx }}(\lambda)=S_{\text {speak }} \frac{W(\lambda, 6000)}{\max [W(\lambda, 6000)]} .
$$

The spectral range of the receiver $E_{\mathrm{det}}$ is given by [9]

$$
E_{\text {det }}(\lambda)=\int_{\lambda_{1}}^{\lambda_{2}} W_{\text {approx }}(\lambda) \mathrm{d} \lambda,
$$

where $\lambda_{1}$ and $\lambda_{2}$ are the boundary values of the optical bandpass filter, and $P_{b g}$ can be obtained as follows [9]:

$$
P_{b g}=E_{\mathrm{det}} T_{0} A \mathrm{n}^{2}
$$

where $T_{0}$ is the peak filter transmission coefficient, which can reach 1, $A$ is the area at the receiving side, and $n$ is the internal refractive index.
Based on the measured global solar irradiance, the trend of $P_{b g}$ during a day can be derived as shown in Figure 1.

As can be seen from Figure 1, before 13:00, with the sun rising, $P_{b g}$ increases from $-3 \mathrm{dBm}(7: 00)$ to $16 \mathrm{dBm}$ (13:00). After 13:00, with the sun falling, $P_{b g}$ decreases to $3 \mathrm{dBm}$ at 18: 30 , and from the above analysis, it is found that $P_{b g}$ changes obviously during a day. Therefore, the influence of background light noise on the performance of visible light communication in fire smoke environment cannot be ignored, and the background light noise caused by sunlight is usually Gaussian white noise, which can be removed by using denoising.

\section{SNR and BER of Visible Light \\ Communication in Fire Smoke Environment}

According to the Lambert-Beer law, when the transmission distance is $D$, the received power $P_{r}$ is determined by the attenuation coefficient. The visibility $(V)$ is obtained from Koschmieder's law [10]:

$$
V=\frac{10 \log _{10}\left(T_{\mathrm{th}}\right)}{\beta_{k}}(\mathrm{~km}),
$$

where $T_{\text {th }}$ is the visual threshold, which usually takes $2 \%$.

The relationship between the attenuation coefficient $\beta_{\lambda}$ and $V$ can be approximated as

$$
V(\mathrm{~km})=\frac{10 \log _{10}\left(T_{\mathrm{th}}\right)}{\beta_{k}}\left(\frac{\lambda}{\lambda_{0}}\right)^{-q},
$$

where $\lambda_{0}$ is the maximum spectral value in the solar band. For the visible wavelength band, it is derived that

$$
\beta_{k}=\int_{380}^{780}-\frac{10 \log _{10}\left(T_{\mathrm{th}}\right)}{V}\left(\frac{\lambda}{\lambda_{0}}\right)^{-q} \mathrm{~d} \lambda .
$$

The coefficient $q$ depends on the experimental data such as the size and distribution of the particles [11]:

$$
q=\left\{\begin{array}{l}
1.6, \quad V>50 \mathrm{~km} \\
1.3, \quad 6 \mathrm{~km}<V<50 \mathrm{~km} \\
0.16 V+0.34, \quad 1 \mathrm{~km}<V<6 \mathrm{~km} \\
V-0.5, \quad 0.5 \mathrm{~km}<V<1 \mathrm{~km} \\
0, \quad V<0.5 \mathrm{~km}
\end{array}\right.
$$

Then $P_{r}$ can be obtained as follows:

$$
P_{r}=\frac{A_{R}}{\pi \theta^{2} L^{2}} P_{\mathrm{t}} e^{-\left(\beta_{\lambda} D\right)}
$$

where $A_{R}$ is the received range, $\theta$ is the beam divergence angle, $D$ is the transmission distance, and $P_{t}$ is the emitted power of the LED. $\beta_{\lambda}$ is the attenuation coefficient $\left(A_{R}=10 \mathrm{~cm}, \theta=60^{\circ}\right.$, and $D=10 \mathrm{~m}$ ).

SNR can be used as a measure of visible light communication's performance, and SNR can be expressed as the relationship between received power and environmental noise, as follows [12]: 


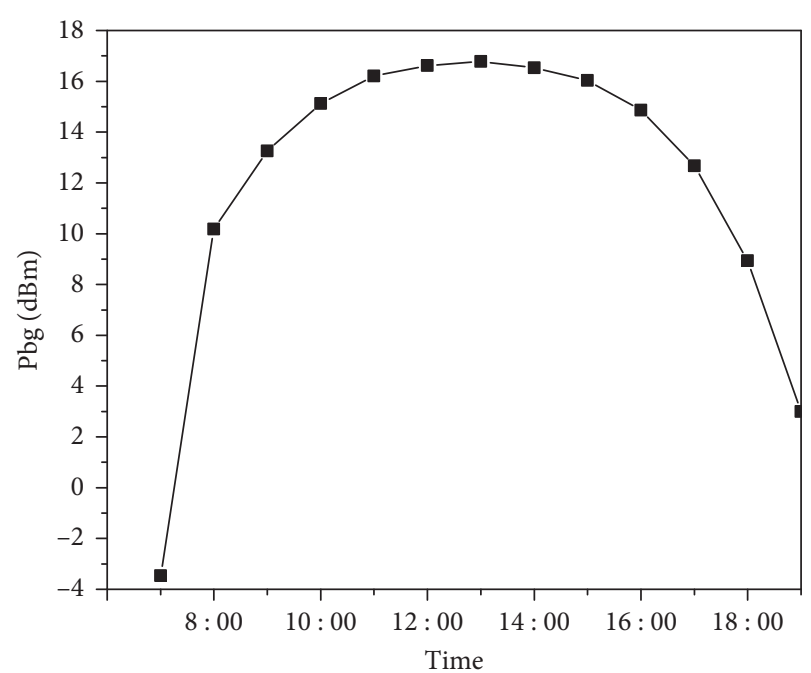

Figure 1: Variation of background light noise during daytime.

$$
\mathrm{SNR}=\frac{R^{2} P_{r}^{2}}{\sigma_{\text {shot }}^{2}+\sigma_{\text {thermal }}^{2}}
$$

where $R$ is the photoresponsivity of the photodetector at the receiver, $\sigma_{\text {shot }}^{2}$ is the scattering noise variance, and $\sigma_{\text {thermal }}^{2}$ is the thermal noise variance.

$$
\sigma_{\text {shot }}^{2}=2 q R P_{r} B+2 q R P_{b g} I_{2} B
$$

where $q$ is electric charge, $B$ is noise bandwidth, and $I_{2}$ is noise bandwidth current [12].

$$
\sigma_{\text {thermal }}^{2}=\frac{8 \pi k T_{k}}{G} \eta A I_{2} B^{2}+\frac{16 \pi^{2} k T_{k} \Gamma}{g_{m}} \eta^{2} A^{2} I_{3} B^{3},
$$

where $k$ is the Boltzmann constant, $T_{k}$ is the absolute temperature, $\eta$ is the capacity per unit area, $A$ is the area at the receiver side, $\Gamma$ is the FET channel noise factor, $G$ is the open-loop voltage gain, $g_{m}$ is the FET transconductance, and $I_{3}$ is the bandwidth coefficient of noise as a percentage of the full ascending cosine.

Using OOK optical intensity modulation, BER can be obtained as follows:

$$
\begin{aligned}
\mathrm{BER} & =\mathrm{Q}(\sqrt{\mathrm{SNR}}), \\
\mathrm{Q}(x) & =\frac{1}{2 \pi} \int_{x}^{\infty} e^{\left(\left(-y^{2}\right) / 2\right)} \mathrm{d} y .
\end{aligned}
$$

Select the LED with $45 \mathrm{~mW}$ emission power; when the background light noise is maximum, the following is $P_{r}$ in different visibility environment.

As can be seen from Figure 2, $P_{r}$ increases with an increase in visibility. $P_{r}$ increases from $0.0122 \mathrm{~mW}(V=1 \mathrm{~m})$ to $0.01302 \mathrm{~mW}(V=12 \mathrm{~m})$ and increases by $6.7 \%$ and finally converges to a constant value of about $0.01302 \mathrm{~mW}$.

The literature [13] gave the smoke attenuation coefficient at different wavelengths, and we took the wavelength of $670 \mathrm{~nm}$ for the calculation and compared it with the measured results in that literature. Figure 3 shows the

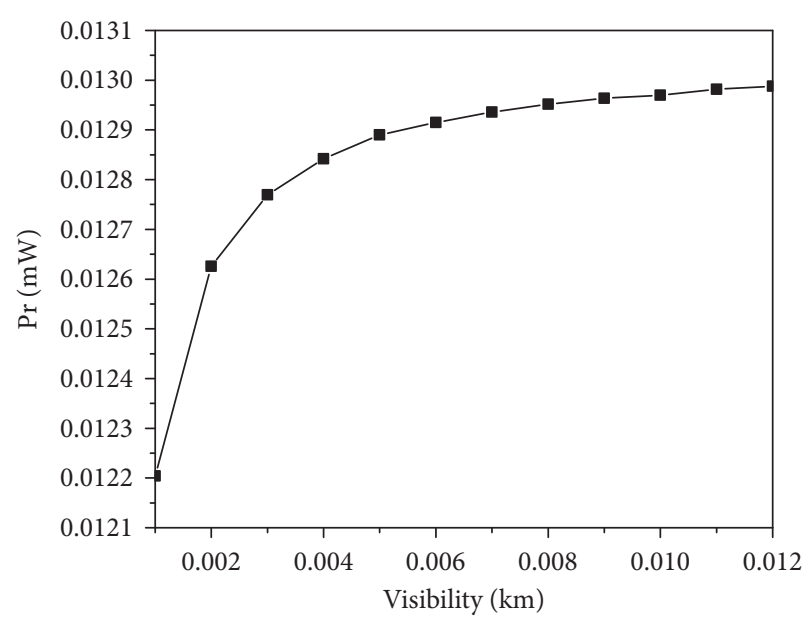

FIGURE 2: Received power in different visibility.

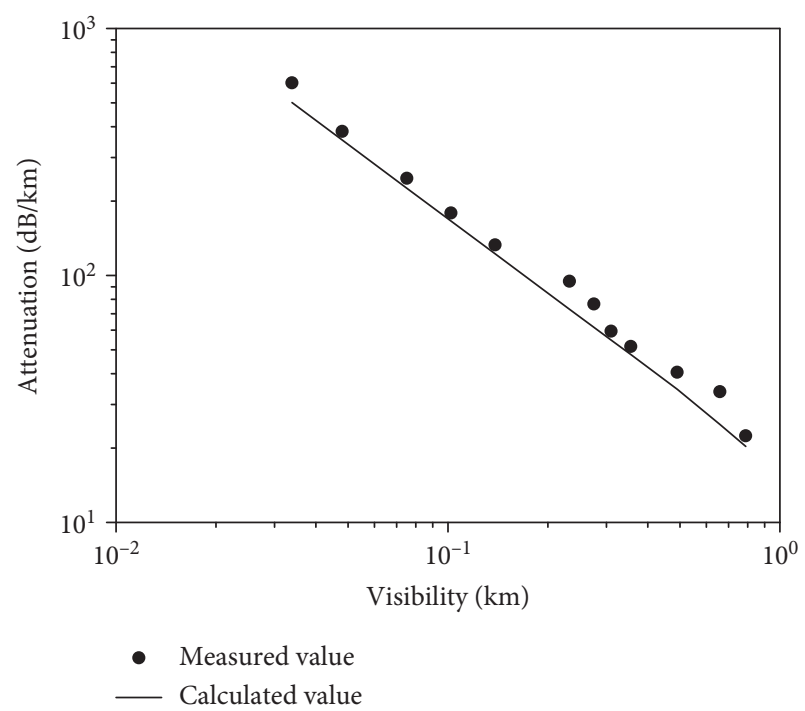

Figure 3: Comparison of the measured value and the theoretical value of the attenuation coefficient.

comparison between the measured and theoretically calculated values of the attenuation coefficient.

As can be seen from Figure 3, the calculated value of attenuation coefficient is basically consistent with the measured value, and the relative error is about $0.07 \%$. Here only the influence of smoke particles and background light noise factors is considered, while the complexity of the actual measurement environment is not only influenced by these two factors but also by the measured attenuation coefficient which is often larger than the theoretical attenuation coefficients. To avoid this problem, sunny weather should be chosen for the actual measurement.

Figures 4 and 5 show the variation of SNR and BER during a day in fire smoke environment with different visibility.

By analyzing Figures 4 and 5, it can be seen that SNR first decreases and then increases, and BER first increases and then decreases during a day. Before 13:00, continuously SNR 


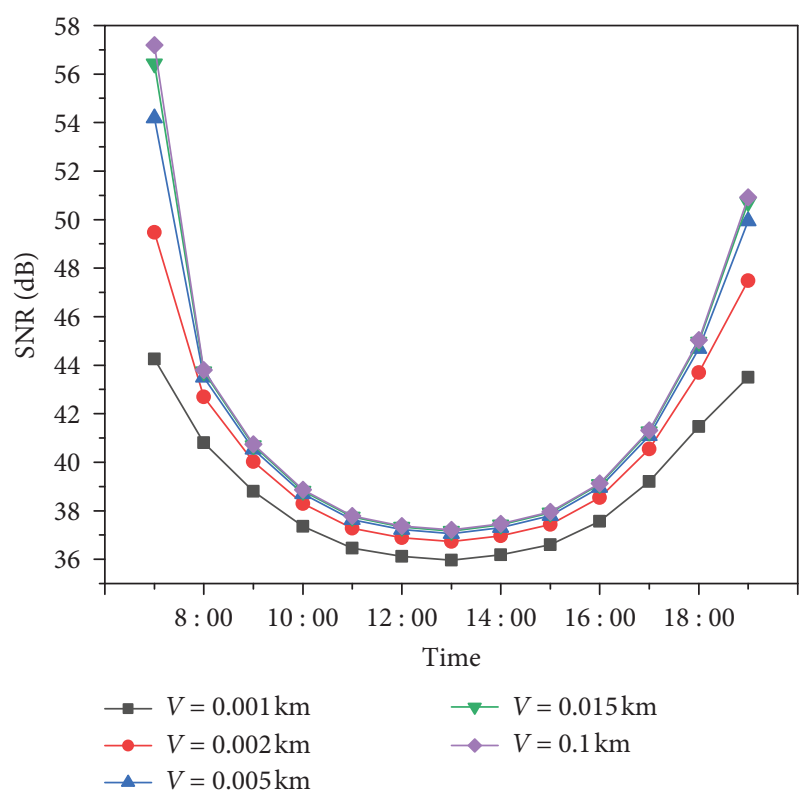

FIGURE 4: SNR for a day in fire smoke environment with different visibility.

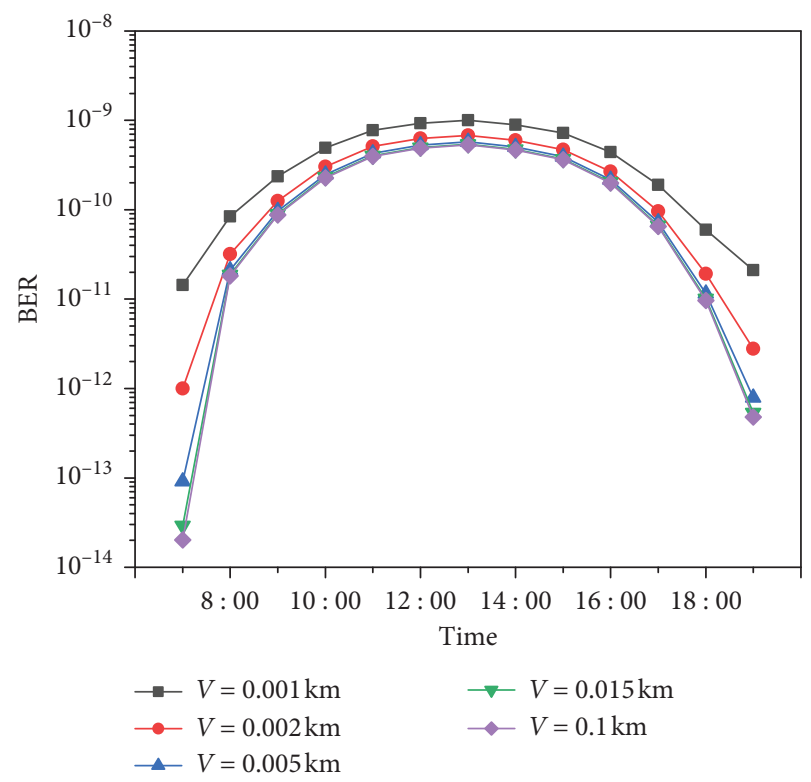

FIgURE 5: BER for a day in fire smoke environment with different visibility.

decreases and BER increases, and finally, SNR reaches the minimum and BER reaches the maximum during a day. After 13:00, SNR increases and BER decreases. In addition, it can be concluded that SNR increases and BER decreases with the increase of visibility.

The visible light communication system can be used to adjust $P_{t}$ to cope with the fire smoke environment. When $V$ is $12 \mathrm{~m}$ and $D$ is $10 \mathrm{~m}, P_{r}$ is calculated to be about $0.013 \mathrm{~mW}$. In order to make the receiving power reach $0.013 \mathrm{~mW}$ at any time, $P_{t}$ can be adjusted according to the real-time visibility.
As shown in Figure 6, when $P_{r}$ is always $0.013 \mathrm{~mW}$, the power of the LED transmitter is adjusted with visibility.

From Figure 6, it can be seen that, in fire smoke environment, when $V$ is less than $0.02 \mathrm{~km}$, the less visibility, the more $P_{t}$ is required, when $V$ is more than $0.02 \mathrm{~km}, P_{t}$ tends to a constant value and is almost no longer affected by visibility. At this time, the transmitter can adjust the value of $P_{t}$ according to different visibility, so that $P_{r}$ is always at $0.013 \mathrm{~mW}$.

\section{Path Loss and Power Loss in Fire Smoke Environment}

Figure 7 shows a simple visible light communication scene in fire smoke environment. The visible light communication link's performance in fire smoke environment is not only affected by the background light noise but also by the absorption of smoke particles.

As can be seen from Figure 7, the distance between the receiving side and the LED is $D$, the radius of the detector at the receiving side is $r$, the angle between the communication link and the normal of the receiving side is $\alpha$, the angle of the communication link to the axis of the LED light beam is $\beta$, $\Omega_{r}$ is the solid angle of the detector's receiving field of view, and the receiving area is $A$. The relationship between them satisfies the following equation [12]:

$$
A \cos \alpha \approx D^{2} \Omega_{r} \text {. }
$$

In the device manual of LED, the axial light intensity $I_{0}$ is usually given, and we can obtain the total luminous flux at the transmitter $F_{s}[12]$ :

$$
F_{s}=\int_{0}^{\Omega_{\max }} I_{0} g_{s}(\theta) \mathrm{d} \Omega=\int_{0}^{\theta_{\max }} 2 \pi g_{s}(\theta) \sin \theta \mathrm{d} \theta,
$$

where $g_{\mathrm{s}}(\theta)$ is the spatial distribution function of the LED lamp and $\Omega_{\max }$ is the stereo angle of the LED beam, which is related to the maximum half-angle of the beam as follows:

$$
\Omega_{\max }=2 \pi\left(1-\cos \theta_{\max }\right) .
$$
[14]:

We can get the total luminous flux at the receiving end $F_{r}$

$$
F_{r}=I_{0} g_{s}(\beta) \Omega_{r}
$$

The LED can be viewed as a Lambertian source, and its spatial distribution function is $g_{s}(\theta)=\cos ^{m}(\theta)$. The Lambert radiation coefficient $m$ can be obtained by $m=$ $(-\ln 2) / \ln \left(\cos \phi_{1 / 2}\right)$ where $\phi_{1 / 2}$ is the radiation half-angle of the LED, and let $\theta_{\max }=\pi / 2$, the path loss $L_{L}$ can be derived by integrating.

$$
\begin{aligned}
L_{L}=\frac{F_{r}}{F_{s}} & =\frac{I_{0} g_{s}(\beta) \Omega_{r}}{I_{0} \int_{0}^{\theta_{\max }} 2 \pi g_{s}(\theta) \sin \theta d \theta}=\frac{g_{s}(\beta) A \cos \alpha}{D^{2} \int_{0}^{\theta_{\max }} 2 \pi g_{s}(\theta) \sin \theta d \theta}, \\
L_{L} & \approx \frac{(m+1) A}{2 \pi D^{2}} \cos \alpha \cos ^{m}(\beta) .
\end{aligned}
$$

In addition, the power loss $L_{P}$ can be expressed as 


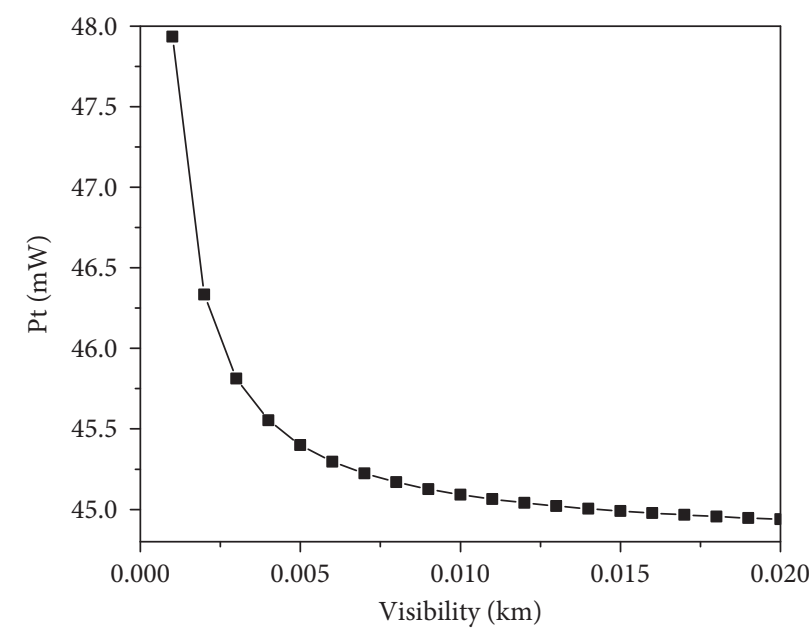

FIGURE 6: Variation of emitted power with visibility.



FIgURE 7: Visible light communication scene in fire smoke environment.

$$
L_{p}=\frac{S_{r}(\lambda)}{S_{s}(\lambda)}
$$

where $S_{s}(\lambda)$ is the power spectral density of the light source and $S_{r}(\lambda)$ is the power spectral density of the receiving side.

According to equation (19), it can be derived that

$$
F=683 \cdot \int_{380 \mathrm{~nm}}^{780 \mathrm{~nm}} S(\lambda) V(\lambda) \mathrm{d} \lambda .
$$

It is known that when the visual acuity function is consistent, $L_{p}=L_{L}$.

LED parameter tables usually give $F_{s}$ or $I_{0}$ of LED, $g_{s}(\theta)$, and the normalized power spectral density $S_{s}^{\prime}(\lambda)$. When $F_{S}$ and $I_{0}$ are interchangeable, $S_{S}(\lambda)$ can be found from $S_{s}(\lambda)=C S_{s}^{\prime}(\lambda)$, where $C$ is the conversion factor, which can be derived from equation (16) [14]:

$$
C=\frac{F_{s}}{683 \times \int_{\lambda_{s L}}^{\lambda_{s H}} S_{s}^{\prime}(\lambda) V(\lambda) \mathrm{d} \lambda},
$$

where $\lambda_{s L}$ and $\lambda_{s H}$ are two endpoint values of the wavelength range of the visible band, respectively, and $S_{r}(\lambda)$ can be obtained by the following formula:

$$
S_{r}(\lambda)=L_{p} S_{s}(\lambda)=C L_{p} S_{s}^{\prime}(\lambda)
$$

An optical filter with the spectral response of $R_{f}(\lambda)$ is added to the photodetector, and $P_{r}$ is expressed as follows:

$$
P_{r}=\int_{\lambda_{r L}}^{\lambda_{r H}} S_{r}(\lambda) R_{f}(\lambda) \mathrm{d} \lambda
$$

where $\lambda_{r L}$ and $\lambda_{r H}$ are two end values of the optical filter [14].

The normalized radiation power spectral density waveforms and visible light filter waveforms are shown in Figures 8 and 9 .

As can be seen from Figure 8, there are two peaks in the normalized radiation power spectrum density of the LED, which are distributed at $450 \mathrm{~nm}$ and $560 \mathrm{~nm}$. LED is a white LED with yellow phosphor on a blue substrate, blue LED itself does not have a very high luminous flux, but after adding the yellow phosphor, the excitation energy is 8 times higher than the original. So this LED is more suitable for visible light communication. As can be seen from Figure 9, the filter has a filter range of $380 \mathrm{~nm}-780 \mathrm{~nm}$, which only allows all wavelengths of visible light to pass through, effectively filtering out the nonvisible band of the spectrum.

$\beta$ is calculated to be constantly changing, when background light noise is maximum, and Figure 10 shows the relationship between $P_{r}$ and $\beta$ in fire smoke environment.

As can be seen from Figure 10, $P_{r}$ is gradually decreasing with the increase in $\beta$. When the LED beam axis coincides 


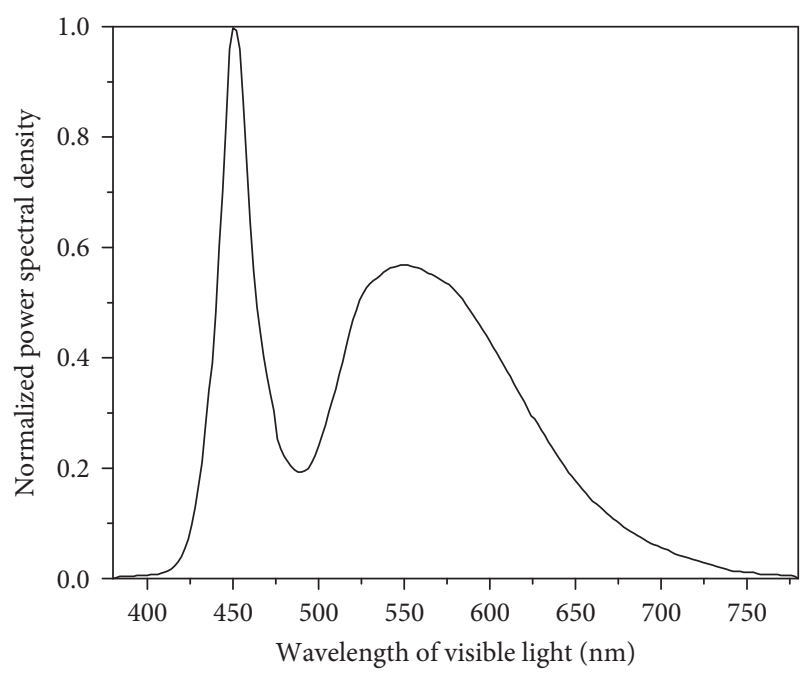

Figure 8: Normalized power spectral density of LEDs.

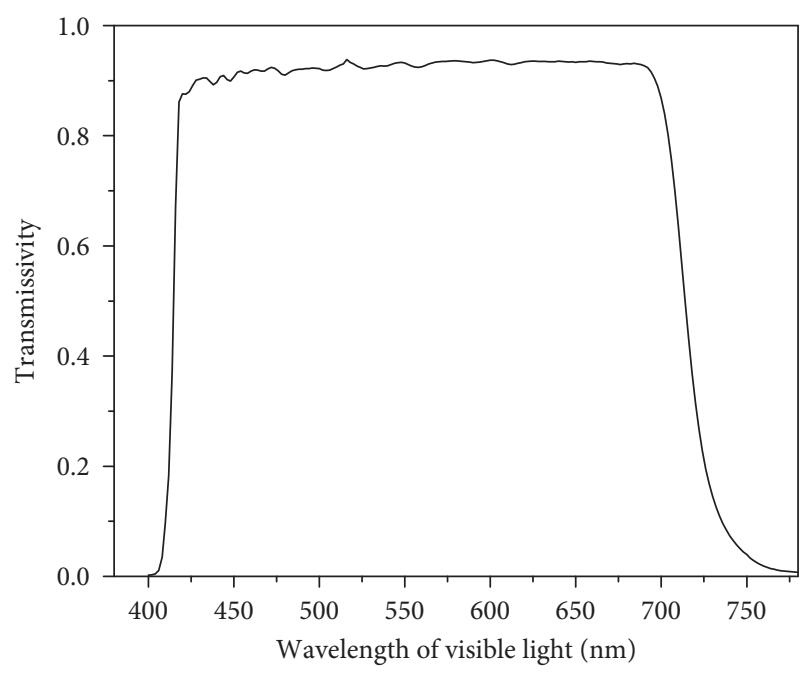

Figure 9: Visible light filter.

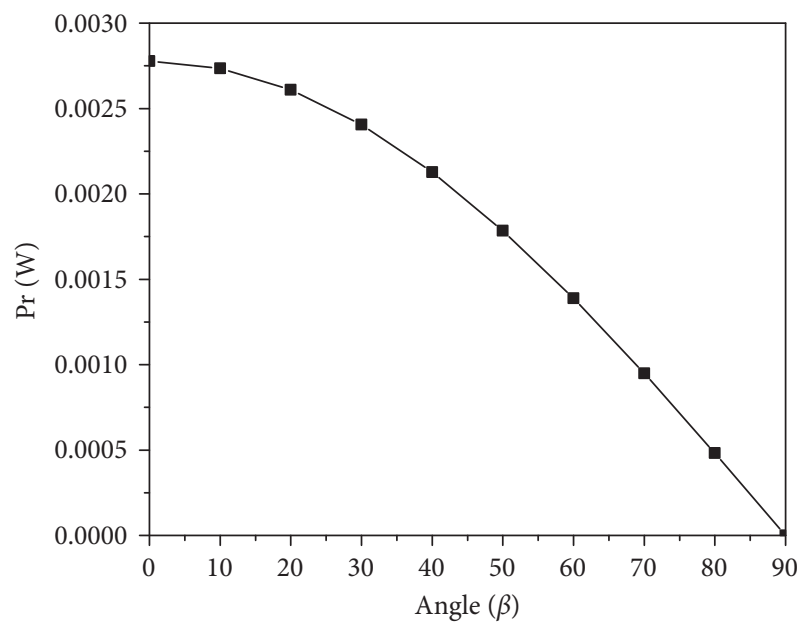

Figure 10: Variation of received optical power with angle $\beta$.
TABLE 1: Parameter table.

\begin{tabular}{lc}
\hline Parameter & Numerical \\
\hline$P_{t}$ & $45 \mathrm{~mW}$ \\
$A$ & $1 \mathrm{~cm}^{2}$ \\
$I_{0}$ & $1100 \mathrm{~cd}$ \\
$N$ & 1.5 \\
$R$ & $0.53 \mathrm{~A} / \mathrm{W}$ \\
$T_{k}$ & $298^{\circ} \mathrm{C}$ \\
$G$ & 10 \\
$g_{m}$ & $30 \mathrm{~ms}$ \\
$\Gamma$ & 1.5 \\
$\mathrm{H}$ & $112 \mathrm{pf} / \mathrm{cm}^{2}$ \\
$I_{2}$ & $0.562 \mathrm{~A}$ \\
$I_{3}$ & $0.0868 \mathrm{~A}$ \\
$B$ & $2 \mathrm{MHz}$ \\
\hline
\end{tabular}

with the communication link, $P_{r}$ can reach its maximum. So in order to improve the communication efficiency of the visible light communication system, it should try to make the LED light axis coincide with the communication link.

\section{Conclusion}

This paper investigates the factors that affect the performance of visible light communication in fire smoke environment. By analyzing the change rule of background light noise during a day, the changing relationship between SNR/ BER and visibility during a day and the influence of $\beta$ was obtained. It is concluded that, with the enhancement of external light, SNR keeps decreasing, BER keeps increasing, and the communication performance decreases. In addition, the received power decreases with the increase in $\beta$.

Through the above study, it is concluded that the effects of fire smoke and background light noise on visible light communication performance cannot be ignored, and it is crucial to improve the performance of LED transmitting equipment and the performance of photoelectric detection equipment at the receiving side. For the transmitter side, the paper gives the corresponding transmitting power according to different visibility, and the adjustment scheme can be applied in the actual fire smoke environment. For the receiver side, in practical applications, the filter can reduce the effect of background light noise by increasing the modulation frequency. Meanwhile, $\beta$ should be minimized. In the next research work, the above scheme will be applied in practice, and in the complex fire environment, the automatic power regulation device is used to verify the above scheme.

Table 1 shows the parameters used in this paper.

\section{Data Availability}

The data used to support the findings of this study are obtained from previous studies $[9,10,12]$.

\section{Conflicts of Interest}

The authors declare that they have no conflicts of interest regarding the publication of this article. 


\section{Acknowledgments}

This work was supported by the National Natural Science Foundation of China (61405157), the National Natural Science Foundation (2019JQ-901), and the Key Industry Innovation Chain Project of Shaanxi Province (2017ZDCXL-GY-06-01).

\section{References}

[1] A. Kumar and V. Sudha, "Optical power distribution and statistical analysis of indoor visible light communication," in Proceedings of the 2019 TEQIP III Sponsored International Conference on Microwave Integrated Circuits, Photonics and Wireless Networks (IMICPW), pp. 383-386, Tiruchirappalli, India, May 2019.

[2] X. Ke, J. Wu, and S. Yang, "Research progress and prospect of atmospheric turbulence for wireless optical communication," Chinese Journal of Radio Science, pp. 1-17, 2021.

[3] Y. Zhu, Y. Zhang, and C. Meng, "Research on the smoke produced by explosion impact on laser transmission," Laser Journal, vol. 34, no. 5, pp. 7-9, 2013.

[4] W. Chen, R. Mu, R. Zhang et al., "Transmittance measurement on smog of solid smoke agent in several wavebands," Journal of Applied Optics, vol. 37, no. 5, pp. 738-741, 2016.

[5] W. Chen, R. Zhang, S. Chen, Y. Duan, S. Ma, and X. Zhang, "Study on multi-wavelength laser attenuation influenced by missible tail smoke," Journal of Applied Optics, vol. 37, no. 5, pp. 772-775, 2016.

[6] X. Wang and J. Duan, "Experiment on polarized laser energy transmission under smoke environment," in Proceedings of the 2015 International Conference on Optoelectronics and Microelectronics (ICOM), Changchun, China, July 2015.

[7] Z. Jiang and C. Song, "The simulation of FMCW laser transmission in smoke environment," in Proceedings of the 2019 4th International Conference on Control and Robotics Engineering (ICCRE), Nanjing, China, April 2019.

[8] I. E. Lee, F. W. L. Kung, and M. L. Sim, "Performance enhancement of outdoor visible-light communication system using selective combining receiver," IET Optoelectronics, vol. 3, no. 1, pp. 30-39, 2009.

[9] S.-J. Lee and S.-Y. Jung, "A SNR analysis of the visible light channel environment for visible light communication," in Proceedings of the 2012 18th Asia-Pacific Conference on Communications (APCC), pp. 709-712, Jeju, Republic of Korea, October 2012.

[10] M. Ijaz, Z. Ghassemlooy, H. L. Minh, S. Rajbhandari, and J. Perez, "Analysis of fog and smoke attenuation in a free space optical communication link under controlled laboratory conditions," in Proceedings of the 4th International Workshop on Optical Wireless Communications, IWOW 2015, Istanbul, Turkey, September 2015.

[11] P. Yang, X. Li, M. Hu et al., "Analysis and calculation of atmospheric transmission characteristics of visible light," in Proceedings of 2007 annual meeting of Shanghai Laser society C, vol. 2, Shanghai, China, 2007.

[12] Y. He, Design and Implementation of Real-Time Radio and Video Visible Light Communication System Based on USB Interface, Nanjing University of Posts and Telecommunications, Nanjing, Jiangsu, China, 2014.

[13] M. Ijaz, Z. Ghassemlooy, J. Pesek, O. Fiser, H. Le Minh, and E. Bentley, "Modeling of fog and smoke attenuation in free space optical communications link under controlled laboratory conditions," Journal of Lightwave Technology, vol. 31, no. 11, pp. 1720-1726, 2013.

[14] Y. Yang, B. Liu, J. Zhang et al., "A visible light communication system based on high-power illumination LED lamp," Journal of Optoelectronics, vol. 22, no. 6, pp. 803-807, 2011. 